\title{
Adaptive Electronic Quizzing Method for Introductory Electrical Circuit Course
}

\author{
doi:10.3991/ijoe.v5i3.930 \\ Ruba A. Amarin, Feras Batarseh and Issa Batarseh \\ University of Central Florida, Orlando, USA
}

\begin{abstract}
The interactive technical electronic book, TechEBook, currently under development at the University of Central Florida, provides a useful tool for engineers and scientists through unique features compared to the most used traditional electrical circuit textbooks available in the market. TechEBook has comprised the two worlds of classical circuit books and an interactive operating platform such as laptops and desktops utilizing Java Virtual Machine operator. The TechEBook provides an interactive applets screen that holds many modules, in which each had a specific application in the self learning process.
\end{abstract}

This paper describes one of the interactive techniques in the TechEBook known as, QuizMe, for evaluating the readers' performance and the overall understanding for all subjects at any stage. The QuizMe will be displayed after each section in the TechEBook for the user to evaluate his/her understanding, which introduces the term me-learning, as a comprehensive full experience for self or individualized education.

In this paper, a practical example of applying the QuizMe feature is discussed as part of a basic electrical engineering course currently given at the University of Central Florida.

Index Terms-QuizMe, Tool, Electrical Circuits, Interactive book.

\section{INTRODUCTION}

The interactive TechEBook consists of 16 chapters, a total of 75 sections representing typical content for the introductory circuit course at most universities. Each section discusses a new theory and concept that are supported with examples and problems [1]. Different topics are presented in discussion text material that provides full understanding of the concept. At the end of each section, QuizMe modules are provided to quiz the students' understanding of the section. Also, TutorMe, modules are part of each section, that are intended to help understanding basic concepts in a step-by-step manner [2]. The Design and the Practical Relevance modules are set to enhance the student thinking about real life problems and also teach students how to relate the theories they have learned with practical applications.

QuizMe was built not only to assess students' understanding of the main concepts, but to help instructors in collecting some cumulative database about their students' performance. This is all done by using software that was specially developed for this application, using Java programming language. QuizMe gives instructors, in any educational institution, a full control in terms of the ability to add, edit or delete questions and their answers, in addition to monitor the overall performance of the students by referring to the database that includes the answers. The different project components are described next.

\section{The Methods RePresented IN QuizME}

\section{A. Project Challenges}

The different natures and types of problems provided in QuizMe create an engaging laboratory environment for students. The variety of the problems given in each different section is meant to test the students' understanding in the classroom through four different difficulty levels. Each question has four properties; the hardness level that reflects the difficulty level of the question, the different groups that categorizes questions into symbolic or numerical questions, the collection, concept, where for each topic there are a group of concepts that the instructors need to address, so they make sure that at least one question is selected in the quiz from each concept, and at last the time can be controlled by the instructor for each question.

These properties are also meant to challenge students from different backgrounds and levels of understanding. Students only interested in understanding the general concept without going deep in solving more enhanced examples can still do this through the first two levels of difficulties. The different types of questions provided by QuizMe enhance the learning process by defining the competencies and deficiencies of the individual being tested. They also identify specific problem areas and needs, through evaluating the individual's performance in each key concept.

\section{B. Project Components}

The learning process is measured by the student achievements, which can be evaluated as accomplished successfully or not through the feedbacks on the quiz at the end of each section. The four different flavors provided by QuizMe is the main aspect in the enhancement of student achievement. It also provides the instructor with a unique teaching strategy that allows the students to start with understanding the concept of a certain section (levels 1 and 2) and then move on to solving more challenging problems (levels 3 and 4). At the end of each QuizMe section, the instructors can review feedback reports which will provide information on the level of understanding and cumulative statistics that the students gained from that section. 


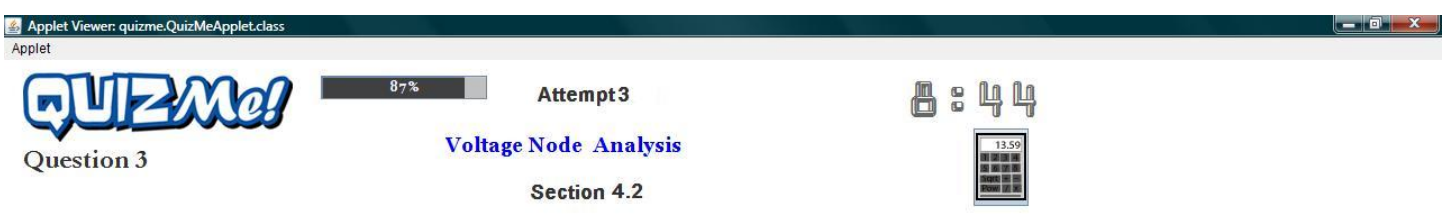

The correct KCL equation applied at nodal voltage vb is given by:

$$
\begin{aligned}
& \text { A) } \frac{v_{a}-v_{b}}{R_{2}}-\frac{v_{b}}{R_{3}}-\frac{v_{b}-v_{c}}{R_{1}}=0 \\
& \text { B) } \frac{v_{a}-v_{b}}{R_{1}}-\frac{v_{b}}{R_{3}}+\frac{v_{c}-v_{b}}{R_{2}}=0 \\
& \text { C) } \frac{v_{a}-v_{b}}{R_{1}}-\frac{v_{b}}{R_{3}}-\frac{v_{c}-v_{b}}{R_{2}}=0 \\
& \text { D) } \frac{v_{b}+v_{a}}{R_{1}}-\frac{v_{b}}{R_{3}}+\frac{v_{c}+v_{b}}{R_{2}}=0
\end{aligned}
$$

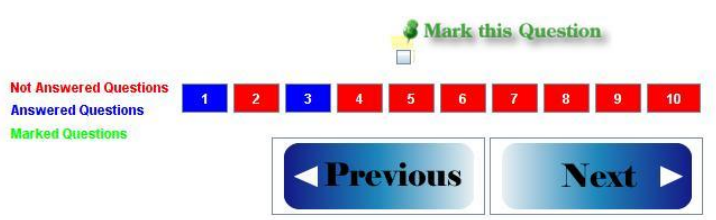

Applet started.

Figure 1. JPanel question

\section{Project Features}

The quality feature is achieved through the unique overall set of problems provided which adds a distinguishing characteristic to the EBook. The applicability is addressed through the different set of problems provided and finally the impact on the learning process can be measured by the students' overall understanding. The big pool of questions for each section is meant to keep students focused on each subject individually until they grasp the needed understanding for that part. Students will be able to test their understanding by taking quizzes and evaluating their performance in each section. The main outcome will be better quality students through a studies end-to-end learning process.

\section{Problems Writing, Tool Modules AND SELECTION CRITERIA}

The quiz at the end of each section contains ten problems selected from a pool of problems saved in the database. Most questions are associated with a circuit image and multiple answer choices in which one is correct. Different values for questions that have numerical representation potential are also in the database. The program randomly picks a number for each component within a certain range and calculates the correct answer along with other multiple wrong answers. The student can take the quiz multiple times. The QuizMe software is programmed in a way that at each time the student takes the quiz, new problems and new values of the components are selected. In another words, no similar numerical problem can appear twice to the same student.

Next, the different classes developed for QuizMe purposes are described. The JAppletQuiz is an extension class of the java's JApplet and is the main container of all the software objects. The JPanelQuestion on the other hand is an extended class of the java swing object JPanel, and is the main screen that the software displays for students [3]. The JPanelQuestion has the questions and all the related data that includes the circuit image, calculator, and timer. Students are given the option to toggle between questions either by moving forward or backward, or by referring to the questions indication panel with the key color code. Students will be able to see what questions did they answer already, which ones are marked and which ones are not answered yet. JPanelQuestion interface is shown in Fig. 1.

The final report will be a screen display that allows students to view the right and wrong answers, grades, and the opportunity to retake the quiz for the same section.

The Editors view and the Managers view are the interfaces for the instructors to edit, modify and load the questions. In the Editors view, instructors can comment on questions (ex: for teaching assistants to modify), add questions, and even see all the images attached to every question and modify the different answers. The Managers view is where the instructors can modify the selection criteria, decide how many numerical questions to have, and put certain constraints on the quiz for better student evaluation. The Editors view is shown in Fig. 2.

The students' performance information data like grades, number of quiz attempts and sections already tested are saved in the program in a class specially developed called the QuizState. Finally, class DBConn is the program's interface with the database, where questions are selected according to the selection criteria, edited, modified and deleted from the data base. 


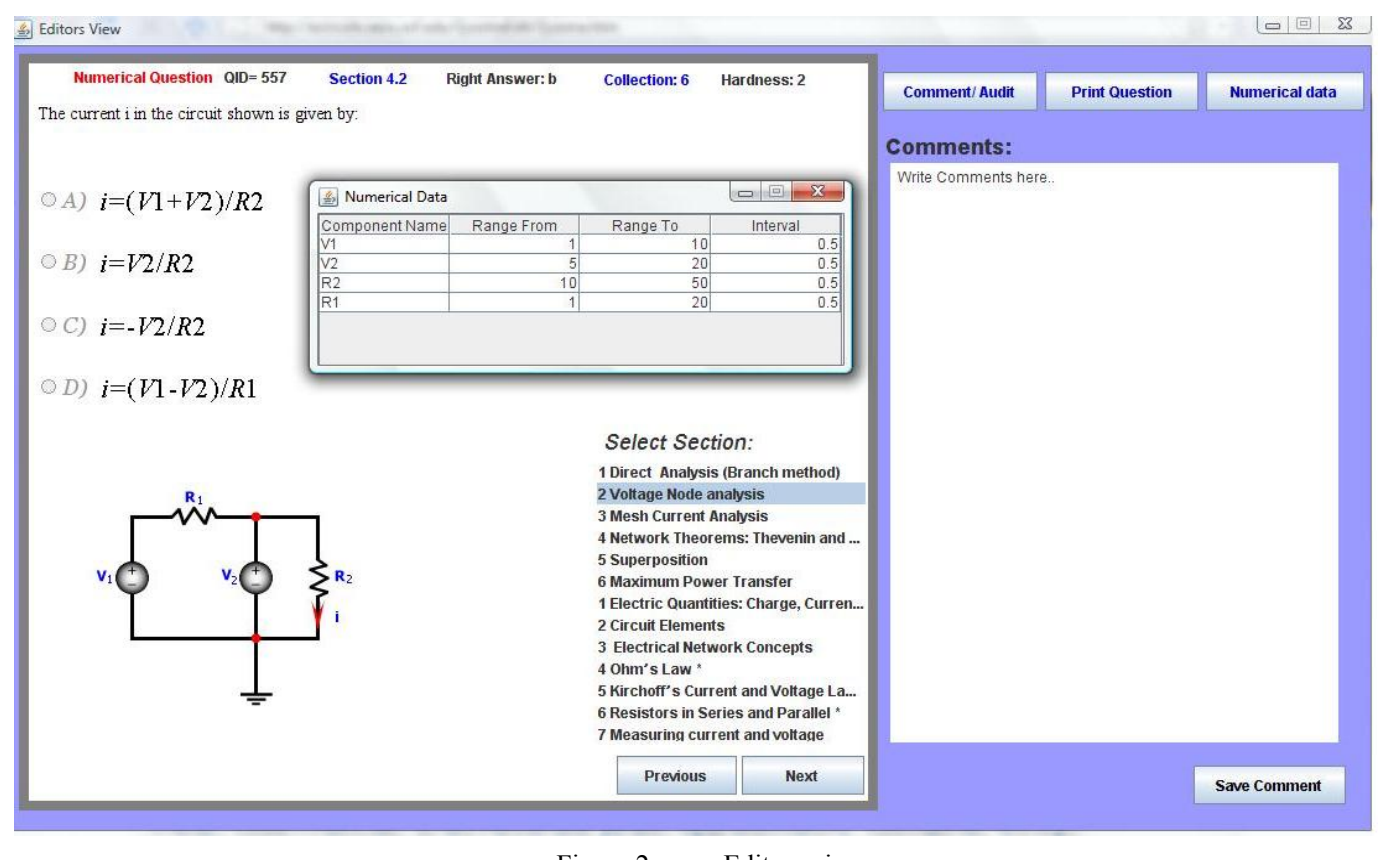

Figure 2. Editors view

The QuizMe tool was evaluated by comparison to some other tools used for the same purpose. As a result, all the other tools offer traditional testing environment, without giving the students the opportunity to use the features proposed in the QuizMe tool. These features include the multiple attempts provided for students to enhance their understanding by giving them the opportunity to solve more questions. The QuizMe tool also adapt itself with the readers' understanding level by increasing the difficulty level of questions each time the reader gets the correct answer but stays at the same level if it was answered wrong. Fig. 3 shows three different circuit hardness levels increasing from left to right, respectively. The level of hardness selection criteria is summarized in Fig. 4. The student starts the quiz with level one difficulty. After each exam attempt, the program estimates the grade based on the answers. If the student wishes to retake the exam, two status checks take place; when the grade is eight or more; the next exam will increase the level of hardness, but if the grade was more than six, then the next exam will display a mixture of the current hardness level and the next. If the grade was less than six, the student will have the same hardness level exam repeated again. The selection criterion is based on choosing one question per collection for each quiz, with the same hardness. Whenever a question is selected, the class DBConn places a flag on the question in the database, to indicate that it has been selected and to avoid selecting it again within the same Quiz.

\section{EXPERIMENTAL RESULTS}

A survey of 54 students of different majors from the Principle in Electrical Engineering (EGN 3373) class at the University of Central Florida was conducted and results were analyzed. $26 \%$ of the students believe that the quiz is a good tool for improving the understanding of the concepts in the class, while $37 \%$ think that their understanding have been improved after using the software. On the other hand, $24 \%$ reported that the quiz was not the only reason behind the understanding of the concepts but the method is good and user friendly, and $11 \%$ reported that the quiz was not effective for them. Fig. 5 reports the two survey questions about the clarity of questions and the used software. Most answers came positive, and the students expressed their willingness to use this tool for all their courses.

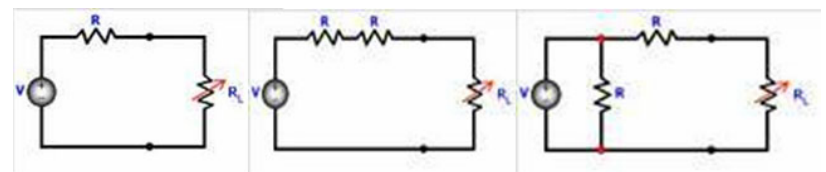

Figure 3. Different hardness circuits for the same concept

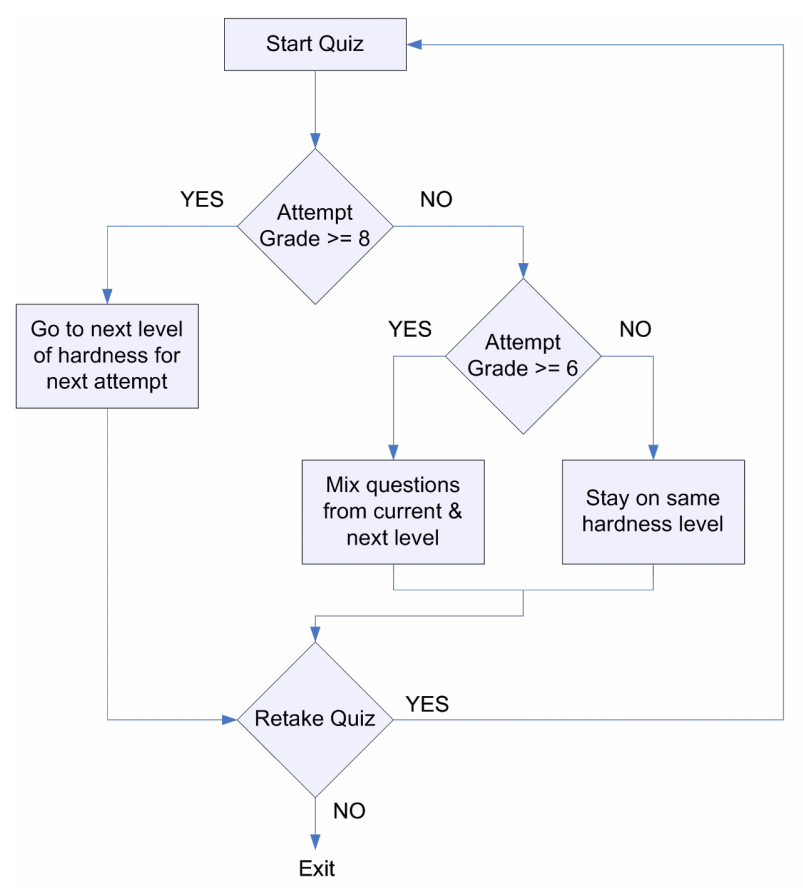

Figure 4. Hardness selection criteria 

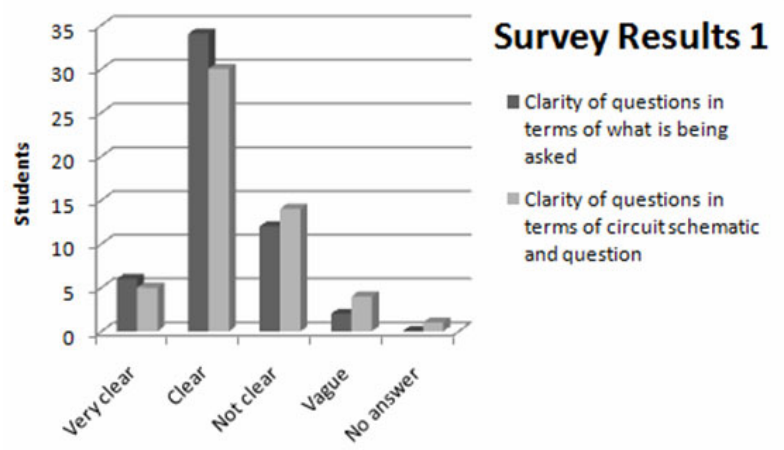

Figure 5.

\section{CONCLUSIONS}

There is no doubt that learning is the world's new language of communication and knowledge delivery, and TechEBook has been used to translate streaked old circuit basic books to a new well developed and easy to use tool. This paper, addresses the TechEBook friendly environment, QuizMe, feature in particular. It presents a new electronic, interactive and adaptive method to maximize the students learning experience, in their self learning process (me-learning) and increase their understanding in any given topic.

The main purpose of the QuizMe technique is to give a well established and fair feedback, and give different ways on how to improve in any certain subject. The overall assessment of 54 students who took the QuizMe module resulted in nearly $79 \%$ of students welcoming the new tool which reflects the importance of this on-line quiz tool.

\section{REFERENCES}

[1] Issa Batarseh, Ghaith Haddad, Rawad Haddad and Rashad Oreifij, 'Interactive Electronic Book Operating Systems And Methods,' United States Patent 20080222552.

[2] Ghaith Haddad, Gustavo Gamboa and Issa Batarseh, 'Interactive Electrical Circuit Tutoring Tool. eTutor,' 2008 ASEE Southeast Section Conference, Memphis, TN, April 2008.

[3] Feras Batarseh, Moataz Abdelwahab and Issa Batarseh, 'Quiz MeAn Interactive Learning Tool with Application to Electrical Circuits,' ASEE SE Section Annual Conference - Marietta, GA, April 2009 .

\section{AUTHORS}

Ruba A. Amarin is with the University of Central Florida (School of Electrical Engineering and Computer Science), Orlando, FL 32816 USA (e-mail: ramarin@mail.ucf.edu). Ruba received the B.S. degree in Electronic Engineering from the Princess Sumaya University

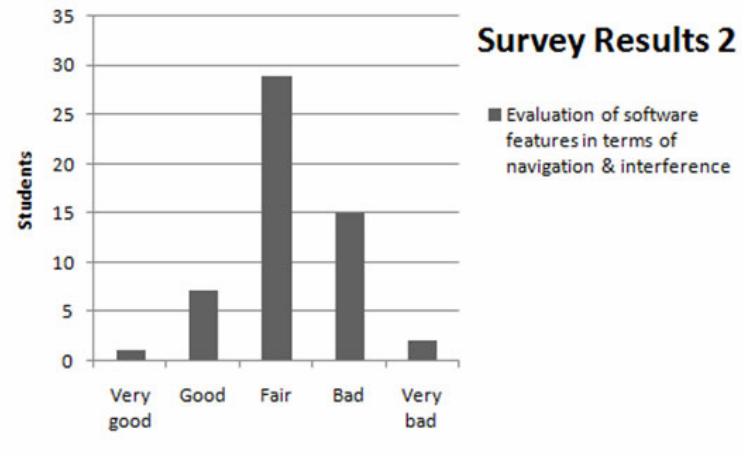

Conducted survey

for Technology, Amman-Jordan in 2004; she afterwards received her M.Sc. degree in Electrical Engineering, TeleCommunication track from the University of Central Florida, Orlando in 2006. Ruba is currently pursuing her Electrical Engineering $\mathrm{PhD}$, conducting research in Satellite and Remote Sensing under the supervision of Dr. Linwood Jones.

Feras Batarseh is with the University of Central Florida (School of Electrical Engineering and Computer Science), Orlando, FL 32816 USA (e-mail: feras_batarseh@yahoo.com). Feras received the B.S. degree in Computer Science from the Princess Sumaya University for Technology, Amman-Jordan in 2006; he afterwards received his M.Sc. degree in Computer Engineering, Software Engineering track from the University of Central Florida, Orlando in 2007. He is currently pursuing his Computer Engineering $\mathrm{PhD}$, conducting research in Software Engineering under the supervision of Dr. David Workman.

Issa Batarseh is a Professor and Director of the School of Electrical Engineering and Computer Science, University of Central Florida, Orlando.32816 USA (e-mail: batarseh@mail.ucf.edu). He received the B.S. degree in Computer Engineering and the M.S. and Ph.D. degrees in Electrical Engineering from University of Illinois, Chicago, in 1983, 1985, and 1990, respectively. He was a Visiting Assistant Professor of Electrical Engineering at Purdue University, Calumet City, IN, from 1989 to 1990 before joining the Department of Electrical and Computer Engineering at the University of Central Florida, in 1991. He has more than 14 U.S. patents, and more than 50 refereed journal and 200 conference publications.

Submitted, May, 15, 2009. Published as resubmitted by the authors on May, 18, 2009. 